\title{
Effect of a selective neutrophil elastase inhibitor on mortality and ventilator-free days in patients with increased extravascular lung water: a post hoc analysis of the PiCCO Pulmonary Edema Study
}

\author{
Takashi Tagami ${ }^{1,2^{*}}$, Ryoichi Tosa ${ }^{3}$, Mariko Omura ${ }^{3}$, Hidetada Fukushima ${ }^{4}$, Tadashi Kaneko ${ }^{5}$, Tomoyuki Endo $^{6}$, \\ Hiroshi Rinka ${ }^{7}$, Akira Murai ${ }^{8}$, Junko Yamaguchi ${ }^{9}$, Kazuhide Yoshikawa ${ }^{10}$, Nobuyuki Saito $^{11}$, Hideaki Uzu ${ }^{12}$, \\ Yoichi Kase $^{13}$, Makoto Takatori ${ }^{14}$, Hiroo Izumino ${ }^{15}$, Toshiaki Nakamura ${ }^{16}$, Ryutarou Seo ${ }^{17}$, Yasuhide Kitazawa ${ }^{18}$, \\ Manabu Sugita ${ }^{19}$, Hiroyuki Takahashi ${ }^{20}$, Yuichi Kuroki ${ }^{21}$, Takayuki Irahara ${ }^{22}$, Takashi Kanemura ${ }^{23}$, \\ Hiroyuki Yokota ${ }^{1}$ and Shigeki Kushimoto ${ }^{24}$
}

\begin{abstract}
Background: Neutrophil elastase plays an important role in the development and progression of acute respiratory distress syndrome (ARDS). Although the selective elastase inhibitor, sivelestat, is widely used in Japan for treating ARDS patients, its effectiveness remains controversial. The aim of the current study was to investigate the effects of sivelestat in ARDS patients with evidence of increased extravascular lung water by re-analyzing a large multicenter study database.
\end{abstract}

Methods: A post hoc analysis of the PiCCO Pulmonary Edema Study was conducted. This multicenter prospective cohort study included 23 institutions in Japan. Adult mechanically ventilated ARDS patients with an extravascular lung water index of $>10 \mathrm{~mL} / \mathrm{kg}$ were included and propensity score analyses were performed. The endpoints were 28-day mortality and ventilator-free days (VFDs).

Results: Patients were categorized into sivelestat $(n=87)$ and control $(n=77)$ groups, from which 329 inverse probability-weighted group patients (162 vs. 167) were generated. The overall 28 -day mortality was 31.1\% (51/164). There was no significant difference in 28-day mortality between the study groups (sivelestat vs. control; unmatched: $29.9 \%$ vs. $32.5 \%$; difference, $-2.6 \%, 95 \%$ confidence interval (Cl), -16.8 to 14.2 ; inverse probability-weighted: $24.7 \%$ vs. $29.5 \%$, difference, $-4.8 \%, 95 \% \mathrm{Cl},-14.4$ to 9.6$)$. Although administration of sivelestat did not alter the number of ventilator-free days (VFDs) in the unmatched (9.6 vs. 9.7 days; difference, $0.1,95 \% \mathrm{Cl},-3.0$ to 3.1 ), the inverse probability-weighted analysis identified significantly more VFDs in the sivelestat group than in the control group (10.7 vs. 8.4 days, difference, $-2.3,95 \% \mathrm{Cl},-4.4$ to -0.2 ).

(Continued on next page)

\footnotetext{
* Correspondence: t-tagami@nms.ac.jp

'Department of Emergency and Critical Care Medicine, Nippon Medical School, 1-1-5 Sendagi, Bunkyo-ku, Tokyo 113-8603, Japan

${ }^{2}$ Department of Clinical Epidemiology and Health Economics, School of Public Health, Graduate School of Medicine, The University of Tokyo, Tokyo, Japan

Full list of author information is available at the end of the article
} 
(Continued from previous page)

Conclusions: Although sivelestat did not significantly affect 28-day mortality, this treatment may have the potential to increase VFDs in ARDS patients with increased extravascular lung water. Prospective randomized controlled studies are required to confirm the results of the current study.

Keywords: Acute lung injury, Extravascular lung water, Pulmonary edema, Pulmonary vascular permeability index, Transpulmonary thermodilution technique

\section{Background}

Acute respiratory distress syndrome (ARDS) is a rapid, progressive form of respiratory failure characterized by life-threatening hypoxemia and permeability pulmonary edema [1-3]. Several previous studies have suggested that neutrophil elastase (NE) increases pulmonary vascular permeability, causes proteolysis of pulmonary tissue, and increases production of leukocyte chemotactic factors, which synergistically cause lung injury $[1,4]$. Previous studies have shown that plasma NE levels correlate with the severity of lung injury in both animal models and human ARDS patients [4,5]. Thus, antagonists or inhibitors of NE synthesis could be effective for treating ARDS. Sivelestat sodium hydrate (Elaspol, Ono Pharmaceutical Co., Ltd., Osaka, Japan) is a specific small-molecule NE inhibitor drug. The effects of this NE inhibitor were demonstrated in various experimental animal models of ARDS [4,6] and also supported by clinical trials [7]. Thus, sivelestat has been approved in Japan and Korea.

However, the effectiveness of sivelestat remains controversial, despite being widely used in Japan for the treatment of ARDS. Two major phase 3 sivelestat trials have been reported to date [8,9]. Tamakuma et al. [9] reported that in a multicenter clinical study, sivelestat contributed to early ventilator weaning in ARDS patients, resulting in earlier transfer to a general ward. However, in the Sivelestat Trial in ALI Patients Requiring Mechanical Ventilation (STRIVE) study [8], sivelestat was not efficacious in patients with ARDS, even in the ventilatorassisted period, and it had no effect on mortality. More recently, positive results were reported following a phase 4 study (postmarket clinical study) in Japan, where both ventilator-free days (VFDs) and 180-day survival rates improved in ARDS patients receiving sivelestat [10]. In contrast, a systematic review and meta-analysis by Iwata et.al [11] concluded that sivelestat was not associated with decreased mortality in ARDS patients. Therefore, although the latest Japanese guidelines for the management of sepsis suggested that sivelestat administration "may be considered" in patients with ARDS [12], its effectiveness remains controversial.

The most reliable pathophysiological feature of ARDS is the development of diffuse alveolar damage (DAD) with increased permeability [13], which results in the accumulation of water in the lungs; this is designated extravascular lung water (EVLW). Although it was difficult to evaluate pulmonary edema caused by acute lung injury (i.e., DAD) quantitatively, recent studies have suggested that transpulmonary thermodilution-derived variables, namely the EVLW index (EVLWi) and the pulmonary vascular permeability index (PVPI), may be informative [14]. Several clinical studies conducted with ARDS patients suggested that both EVLWi and PVPI correlated with disease severity and were independent risk factors of 28-day mortality [15-17]. Thus, these variables have significant clinical implications and may be the key "bridge" for a pathologic-clinical correlation $[14,18]$. However, no previous sivelestat study has measured these indices.

The aim of the current study was to investigate the effect of sivelestat on mortality and VFDs in ARDS patients with evidence of increased EVLW, by re-analyzing a large multicenter cohort study database.

\section{Methods}

The current study was a post hoc analysis of the PiCCO Pulmonary Edema Study, a prospective cohort study that examined patients with respiratory-distress who were admitted to 23 participating institutions in Japan $[16,17,19-22]$. This study was approved by the ethics committee of the Nippon Medical School Hospital, and all the other 22 participating institutional ethics committees, and written informed consent was provided by all of the patients' next of kin.

\section{Data source and patient selection}

The detailed PiCCO Pulmonary Edema study protocols have been described previously [16,17,19-22]. In short, between March 2009 and August 2011, 301 patients with respiratory insufficiency were enrolled in the PiCCO Pulmonary Edema Study. The primary inclusion criteria were as follows: age of $>15$ years, mechanical ventilation expected to be required for $>48 \mathrm{~h}$ for acute respiratory failure with a $\mathrm{PaO}_{2} / \mathrm{FiO}_{2}$ ratio of $\leq 300 \mathrm{mmHg}$, and bilateral infiltration, as determined by chest radiography. An increase in the EVLWi of $>10 \mathrm{~mL} / \mathrm{kg}$ was used to define pulmonary edema, in accordance with published definitions [19]. The exclusion criteria were as follows: a lapse of over 5 days from the onset of acute respiratory failure; chronic respiratory insufficiency; a history of pulmonary 
resection, pulmonary thromboembolism, or severe peripheral arterial disease; a cardiac index of $<1.5 \mathrm{~L} / \mathrm{min} / \mathrm{m}^{2}$; lung contusion; burns; and other causes rendering the patient unsuitable for evaluation with the transpulmonary thermodilution technique [19].

Assessment of the pathophysiological diagnostic differential for respiratory insufficiency was performed by at least three experts (specializing in intensive care, respiratory disease, and cardiology) who retrospectively determined the pathophysiological mechanism of respiratory insufficiency as being (a) cardiogenic (hydrostatic) pulmonary edema, (b) permeability pulmonary edema (i.e., ARDS), or (c) pleural effusion with atelectasis but no evidence of lung edema secondary to increased hydrostatic pressure or vascular permeability as previously described [19]. The experts carefully scrutinized each patient's medical history, clinical presentation, and course and the results of their chest computed tomography, radiography, and echocardiography examinations. They also considered the time course of all of the preceding findings, including daily fluid intake, output, and the balance thereof, and the requirement for systemic management and respiratory therapy.

We considered the increased permeability pulmonary edema group (i.e., (b) above) as ARDS $[16,19]$ and included these patients in the current study. Patients who were not administered sivelestat on Day 0 were excluded from the current analysis. The decision to use sivelestat or not was left to the physician in charge of each participating institution. The standard dosage of sivelestat approved in Japan $(0.2 \mathrm{mg} / \mathrm{kg} / \mathrm{h})$ was administered intravenously after intensive care unit (ICU) admission until it was discontinued based on a clinical decision.

\section{Variables and endpoint}

At the time of enrollment (Day 0), the patient was evaluated with regard to his/her clinical condition, cause of respiratory insufficiency, acute physiology and chronic health evaluation (APACHE) II score, and sequential organ failure assessment (SOFA) score [23,24]. Echocardiography and chest-computed tomography were conducted on the day of enrollment. The patient's clinical course, including respirator setting, SOFA score, daily fluid intake/output and balance, single-indicator transpulmonary thermodilution-derived variables, and therapeutic interventions were recorded daily. The hospital type was categorized as academic or non-academic. Hospital volume was defined as the number of patients that participated in the current analysis and was categorized into tertiles (i.e., low, medium, and high). The principles and validation of the single-indicator transpulmonary thermodilution-derived variables used in the current study have been discussed in detail elsewhere [14]. The EVLW estimates the amount of water present in the lungs and, thus, the extent of pulmonary edema. The PVPI allows for quantitative differentiation of hydrostatic pulmonary edema from ARDS $[19,25]$ and is strongly correlated with the plasma NE level [26]. The PVPI was calculated as the ratio between the EVLW and the pulmonary blood volume. The EVLW was indexed using predicted body weight (EVLWi; normal range $7.4 \pm 3.3 \mathrm{~mL} / \mathrm{kg}$ ) [27-29].

The endpoints used in the current study were 28-day mortality and VFDs [30]. VFDs were defined as the number of days alive and breathing without mechanical assistance during the first 28 days after admission, and patients who died before day 28 were assigned zero VFDs [30].

\section{Statistical analysis}

Data were expressed as mean (standard deviation [SD]) or median (quartile) as appropriate. Continuous variables were compared between the groups using the $t$-test or Mann-Whitney $U$-test as appropriate. Categorical variables were analyzed using the chi-square test or Fisher's exact test. The patients were divided into two groups: those who received sivelestat and those who did not (control group). Descriptive statistics are presented for all patients, propensity score-weighted (inverse probability of treatment weighting, IPTW) groups, and propensity score-matched groups. The usefulness and details of the propensity score approach in clinical studies have been discussed elsewhere $[10,31,32]$. To estimate the propensity score, we fitted a regression model for receipt of sivelestat as a function of patient demographics and hospital factors including age, sex, hospital type (academic or nonacademic), hospital volume, cause of ARDS (direct or indirect lung injury), APACHE II score, SOFA score, systemic inflammatory response syndrome (SIRS) score, mean arterial pressure, central venous pressure, fluid balance on Day $0, \mathrm{PaO}_{2} / \mathrm{FiO}_{2}$ ratio, positive end-expiratory pressure (PEEP) level, global end-diastolic volume index (GEDI) on Day 0, cardiac index on Day 0, EVLWi on Day 0, PVPI on Day 0, corticosteroid administration, catecholamine administration, continuous hemodiafiltration therapy, diuretic administration, and polymyxin B hemoperfusion therapy $[3,7-12,18,23,24,26,33-52]$. Oneto-one matched analysis using nearest-neighbor matching and IPTW estimators were performed based on the estimated propensity scores of the patients. A match occurred when a patient in the sivelestat group had an estimated score within 0.25 standard deviations of a patient in the control group. IPTW used weights based on the propensity score to create a synthetic sample in which the distribution of measured baseline covariates was independent of treatment assignment. Weights were defined as $\mathrm{W} i=\mathrm{Z} i / \mathrm{e} i+(1-\mathrm{Z} i) / 1-\mathrm{e} i$, where $\mathrm{Z} i$ was an indicator variable denoting whether or not the $i$-th subject was treated, and ei denoted the propensity score for the $i$-th subject. An essential component to any propensity 
score analysis is an assessment of the similarity of baseline covariates (i.e., balancing) between treated and untreated subjects in the matched sample or in the sample weighted by IPTW. We examined balance in baseline variables using standardized differences, where $>0.10$ was regarded as imbalanced [31,32]. All data were analyzed using SPSS 22.0 for Windows (IBM, Armonk, NY, USA).

\section{Results}

\section{Patients}

Of the 301 patients initially diagnosed with acute respiratory failure, 192 met the primary inclusion criteria with regard to a diagnosis of ARDS (Figure 1). Of these 192 patients, 164 did not meet any of the secondary exclusion criteria and were evaluated further (Figure 1). These patients were categorized into sivelestat $(n=87)$ and control $(n=77)$ groups, from which 31 propensity score-matched pairs and 329 IPTW patients (162 vs. 167) were generated. Number of patients $(n)$ in the IPTW groups was an "estimated number" determined by weighting inversed probability (i.e., propensity score). Distributions of propensity scores in the unmatched, propensity score-matched, and IPTW groups are shown in Additional file 1: Figure S1.

Table 1 shows the baseline characteristics of the unmatched sivelestat and control groups $(n=164)$, IPTW group $(n=329)$, and propensity score-matched groups

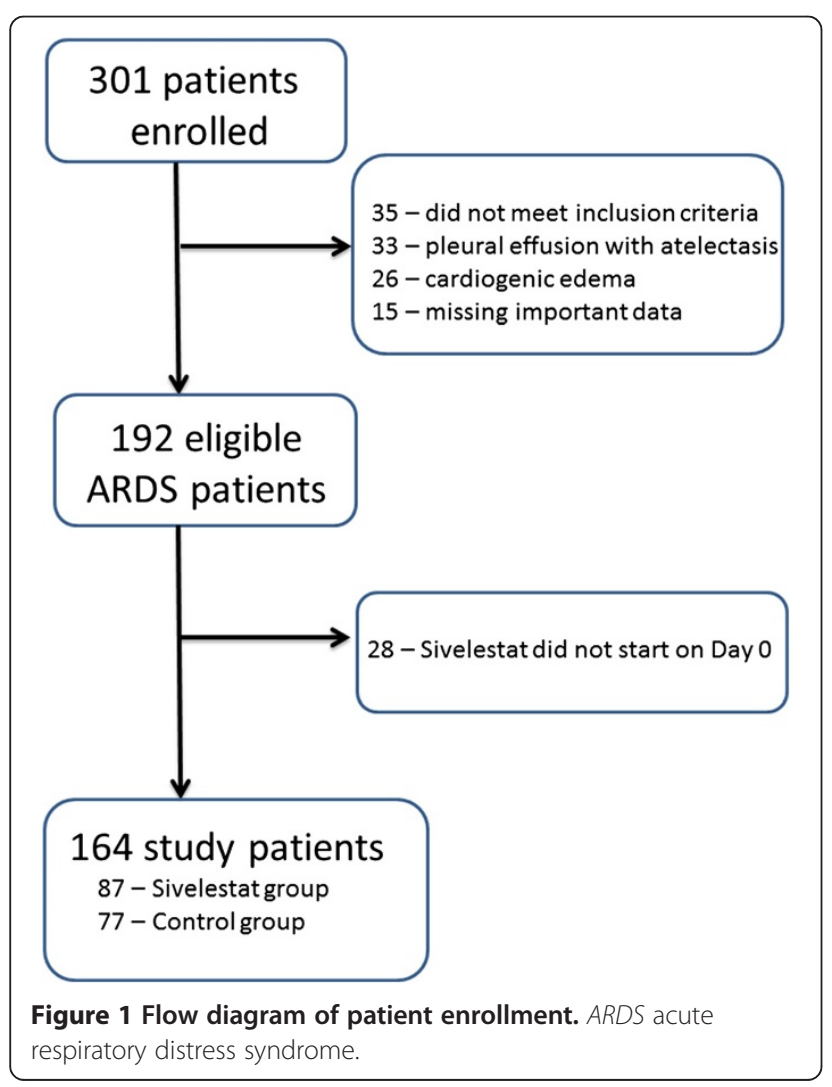

$(n=62)$. When the unmatched groups were compared, patients were more likely to receive sivelestat if they had a positive fluid balance and higher EVLWi on Day 0 . After propensity score IPTW was performed, the baseline patient characteristics were well balanced between the groups. However, we found that the standardized difference of some variables in the propensity-matched groups exceeded 0.10 , suggesting that these were not well balanced. Thus, we decided that it was not appropriate to perform further analysis using the matched pair approach. The median period of sivelestat use was 9 (quartile, 6) days.

\section{Endpoints}

The overall 28-day mortality was $31.1 \%$ (51/164). There was no significant difference in 28-day mortality between the study groups (sivelestat vs. control; unmatched: $29.9 \%$ vs. $32.5 \%$; difference, $-2.6 \%, 95 \%$ confidence interval (CI), -16.8 to 14.2 ; IPTW: $24.7 \%$ vs. $29.5 \%$, difference, $-4.8 \%, 95 \% \mathrm{CI},-14.4$ to 9.6$)$.

Although there was no difference in the number of VFDs in the sivelestat and control groups for unmatched patients ( 9.6 vs. 9.7 days; difference, $0.1,95 \% \mathrm{CI},-3.0$ to $3.1)$, there were significantly more VFDs in the sivelestat group than in the control group for the IPTW (10.7 vs. 8.4 days, difference, $-2.3,95 \% \mathrm{CI},-4.4$ to -0.2$)$.

\section{Discussion}

This retrospective multi-institutional study did not identify any significant association between the 28-day mortality and sivelestat use. However, there may be a weak association between sivelestat use and an increased number of VFDs in ARDS patients with increased EVLW.

Although sivelestat has been approved and is currently used clinically in Japan and Korea, its effectiveness in ARDS patients remains controversial. Previous studies reported conflicting results [7-10,35,36,38,45,47,48,51]. While some reported that sivelestat administration was an independent predictor of survival and contributed to early improvements in oxygenation, early weaning from mechanical ventilation, and early discharge from the ICU $[9,10,35,36,47,48]$, others reported little effect [51] or even negative effects [8].

The strength of the current study was that we took the EVLWi and PVPI into consideration during patient selection and the process used to estimate propensity scores. Previous studies suggested that diffuse alveolar damage resulted in significant accumulation of EVLW in patients with the early phase of ARDS $[3,13,52]$, which led to severe respiratory failure and dependence on mechanical ventilation. Although it is difficult to evaluate the degree of lung injury quantitatively, introduction of the transpulmonary thermodilution technique has facilitated bedside evaluation of the EVLW with robust 
Table 1 Patient characteristics

\begin{tabular}{|c|c|c|c|c|c|c|c|c|c|}
\hline \multirow[b]{2}{*}{ Patient characteristic } & \multicolumn{3}{|l|}{ Unadjusted groups } & \multicolumn{3}{|l|}{ IPTW Groups } & \multicolumn{3}{|l|}{ Matched groups } \\
\hline & Sivelestat $(n=87)$ & Control $(n=77)$ & $\begin{array}{l}\text { Standardized } \\
\text { difference }\end{array}$ & Sivelestat $(n=162)$ & Control $(n=167)$ & $\begin{array}{l}\text { Standardized } \\
\text { difference }\end{array}$ & Sivelestat $(n=31)$ & Control $(n=31)$ & $\begin{array}{l}\text { Standardized } \\
\text { difference }\end{array}$ \\
\hline Age, year & $66.1(17.5)$ & $65.9(16.2)$ & 0.01 & $67.7(16.2)$ & $69.0(15.8)$ & -0.08 & $69.8(15.6)$ & $67.3(15.3)$ & 0.16 \\
\hline Male sex & $57(65.5)$ & $50(64.9)$ & 0.01 & $104(64.2)$ & $114(68.3)$ & -0.09 & $21(67.7)$ & $19(61.3)$ & 0.13 \\
\hline Academic hospital & $49(56.3)$ & $48(63.3)$ & -0.14 & $92(56.8)$ & $86(51.8)$ & 0.10 & $20(64.5)$ & $15(48.4)$ & 0.33 \\
\hline \multicolumn{10}{|l|}{ Hospital volume (cases) } \\
\hline Low (1-10) & $37(42.5)$ & $43(55.8)$ & -0.27 & $73(45.1)$ & $70(41.9)$ & 0.06 & $15(48.4)$ & $14(45.2)$ & 0.06 \\
\hline Medium (11-20) & $19(21.8)$ & $18(23.4)$ & -0.04 & $32(19.8)$ & $34(20.4)$ & -0.01 & $8(25.8)$ & $5(16.1)$ & 0.28 \\
\hline $\operatorname{High}(\geq 21)$ & $31(35.6)$ & $16(20.8)$ & 0.33 & $57(35.2)$ & $63(37.7)$ & -0.05 & $8(25.8)$ & $12(38.7)$ & -0.28 \\
\hline Direct lung injury & $54(62.1)$ & $44(57.1)$ & 0.10 & $94(58.4)$ & $104(62.3)$ & -0.08 & $19(61.3)$ & $21(67.7)$ & -0.13 \\
\hline APACHE ॥ & $22.2(7.4)$ & $23.5(7.4)$ & -0.18 & $23.5(7.3)$ & $23.3(7.5)$ & 0.03 & $23.7(5.5)$ & $24.0(7.6)$ & -0.05 \\
\hline SOFA score & $10.1(3.3)$ & $11.2(3.6)$ & -0.32 & $10.7(3.3)$ & $10.6(3.7)$ & 0.03 & $10.8(3.3)$ & $10.0(3.2)$ & 0.25 \\
\hline SIRS score & $2.3(1.0)$ & $2.4(1.2)$ & -0.09 & $2.4(1.1)$ & $2.4(1.1)$ & 0.00 & $2.4(1.2)$ & $2.5(1.1)$ & -0.09 \\
\hline MAP, mmHg & $75.5(18.3)$ & $77.2(15.5)$ & -0.10 & $77.3(19.1)$ & $78.8(17.2)$ & -0.08 & $75.8(20.1)$ & $73.9(14.3)$ & 0.11 \\
\hline $\mathrm{CVP}, \mathrm{mmHg}$ & $10.7(5.4)$ & $9.3(5.2)$ & 0.26 & $9.8(5.2)$ & $9.7(5.1)$ & 0.02 & $10.5(4.7)$ & $9.5(5.8)$ & 0.19 \\
\hline Fluid balance on Day $0, \mathrm{~mL}$ & $2,239(2,228)$ & $1,498(1,640)$ & 0.38 & $1,915(1,977)$ & $1,861(1,686)$ & 0.03 & $1,495(2,267)$ & $1,673(2,333)$ & -0.08 \\
\hline $\mathrm{PaO}_{2} / \mathrm{FiO}_{2}$ & $148.5(73.1)$ & $148.4(65.6)$ & 0 & $142.9(70.2)$ & $145.6(63.9)$ & -0.04 & $141.7(71.1)$ & $128.5(60.2)$ & 0.20 \\
\hline PEEP, $\mathrm{cm} / \mathrm{H}_{2} \mathrm{O}$ & $8.4(4.9)$ & $8.3(4.1)$ & 0.02 & $8.2(4.6)$ & $8.1(3.8)$ & 0.02 & $8.1(5.0)$ & $8.8(4.6)$ & -0.15 \\
\hline Cardiac index, $\mathrm{L} / \mathrm{m}^{2}$ & $3.5(1.4)$ & $3.5(1.2)$ & 0 & $3.4(1.4)$ & $3.4(1.1)$ & 0.00 & $3.7(1.5)$ & $3.2(1.0)$ & 0.39 \\
\hline GEDI, $\mathrm{mL} / \mathrm{m}^{2}$ & $836.9(236.4)$ & $784.2(158.0)$ & 0.26 & $809.9(208.4)$ & $805.2(149.3)$ & 0.03 & $805.9(167.5)$ & $761.9(150.1)$ & 0.28 \\
\hline EVLWi, mL/kg & $20.0(7.1)$ & $17.2(6.4)$ & 0.41 & $19.1(6.4)$ & $19.4(7.6)$ & -0.04 & $17.1(6.2)$ & $18.5(7.1)$ & -0.21 \\
\hline PVPI & $3.5(1.5)$ & $3.1(1.2)$ & 0.29 & $3.3(1.3)$ & $3.4(1.3)$ & -0.08 & $3.0(0.98)$ & $3.5(1.5)$ & -0.39 \\
\hline Corticosteroid use & $38(43.7)$ & $26(33.8)$ & 0.20 & $62(38.3)$ & $69(41.3)$ & -0.06 & $12(41.9)$ & $10(32.3)$ & 0.20 \\
\hline Catecholamine use & $58(66.7)$ & $59(76.6)$ & -0.22 & $117(72.2)$ & $113(67.7)$ & 0.10 & $23(74.2)$ & $23(74.2)$ & 0.00 \\
\hline Renal replacement therapy & $25(28.7)$ & $18(23.4)$ & 0.12 & $45(27.8)$ & $50(30.1)$ & -0.05 & $9(29.0)$ & $6(19.4)$ & 0.23 \\
\hline Diuretic use & $43(49.4)$ & $38(49.4)$ & 0.00 & $75(46.6)$ & $84(50.3)$ & -0.07 & $16(51.6)$ & $13(41.9)$ & 0.20 \\
\hline PMX use & $12(13.8)$ & $6(7.6)$ & 0.20 & $18(11.1)$ & $14(8.4)$ & 0.09 & $4(12.9)$ & $1(3.2)$ & 0.36 \\
\hline
\end{tabular}

Number of patients $(n)$ in the IPTW groups was an estimated number determined by weighting inversed probability (propensity score).
$A P A C H E$, acute physiology and chronic health evaluation, CVP, central venous pressure, EVLWi, extravascular lung water index, GEDI, global end-diastolic volume index, IPTW, inverse probability of treatment weighting PEEP, positive end-expiratory pressure, PMX, polymyxin B hemoperfusion, PVPI, pulmonary vascular permeability index, SIRS, systemic inflammatory response syndrome, SOFA, sequential organ failure assessment. 
validation $[14,18,25,27-29,53,54]$. We recently validated the clinicopathological relationships between the EVLW and diffuse alveolar damage by conducting pathologic studies and a nationwide autopsy database study [18]. Recent studies showed that the EVLWi reflected the severity of lung injury and correlated with mortality in ARDS patients [15-17,55-57]. In the current study, only ARDS patients with an EVLWi of $>10 \mathrm{~mL} / \mathrm{kg}$ were included, consistent with previous related studies [19]. The normal EVLWi is approximately $7 \mathrm{~mL} / \mathrm{kg}$ [27], and an EVLWi of $>10 \mathrm{~mL} / \mathrm{kg}$ represented the quantitative threshold for the diagnosis of ARDS [18]. Recent studies suggested that the EVLWi (and PVPI) may provide the most reliable characterization of ARDS, where the development of diffuse alveolar damage results in increased permeability and accumulation of water in the lungs $[19,25]$. Thus, EVLWi and PVPI provide key clinical insights into the underlying disease pathology.

The propensity score analysis approach is a powerful tool that attempts to construct a randomized experimentlike situation by comparing groups with similar characteristics without specifying the relationships between confounders and outcomes. In the current study, analysis of the baseline patient characteristics in the unmatched group (the overall study population) showed more sivelestat use in patients with increased EVLWi and positive fluid balance, which are both known to affect outcome in ARDS patients $[2,15]$. We therefore believe that these variables need to be balanced when evaluating the effect of sivelestat on ARDS. In our study, factors that had the potential to affect mortality, or were known to affect mortality in patients with ARDS, were successfully balanced in the IPTW analysis. Our results suggested that administration of sivelestat does not influence 28-day mortality. On the other hand, the IPTW analysis suggested that ARDS patients who were prescribed sivelestat had more VFDs than similar patients who were not. Although we could not draw any robust conclusions regarding the effect of sivelestat in the current retrospective analysis, these results can be considered as hypothesis-generating. Thus, further large prospective trials, considering EVLWi in the entry criteria, are required to confirm the current results.

This study had several limitations. First, the study was conducted retrospectively; it was not randomized or blinded, and the decision regarding administration of sivelestat was made by the doctors in charge of each of the 23 participating institutions. Although propensity score methods were used to adjust for differences in baseline characteristics and disease severity, bias could still be present in the form of confounders that were not measured. Second, even though 164 patients were evaluated in the current study, the nearest-neighbor matching method only identified 31 pairs for one-to-one matched analysis. As a result, the standardized difference of several variables in the propensity-matched groups exceeded 0.10 , suggesting that these were not well balanced. This may be due to the small sample size (31 pairs). In general, pair matching on the propensity score requires that the number of untreated subjects be larger (and preferably substantially larger) than the number of treated subjects [31]. Thus, matching will not perform well when the two samples are of approximately equal size or when the number of treated subjects is larger than the number of untreated subjects [31]. On the other hand, IPTW methods do not suffer from this limitation [31]. One of the strengths of the IPTW method is that data from all of the patients were used. On the other hand, treated subjects with a very low propensity score or untreated subjects with a high propensity score will have large weights, as shown in Additional file 1: Figure S1. This might be a significant concern if the study group was heterogeneous. However, in the current study, we tried our best to select a homogeneous patient group by utilizing strict inclusion and exclusion criteria (i.e., even the control group had the potential to receive sivelestat): all patients were mechanically ventilated in the tertiary care hospital ICU for $>48 \mathrm{~h}$ due to acute respiratory failure, $\mathrm{PaO}_{2} / \mathrm{FiO}_{2}$ ratio of $\leq 300 \mathrm{mmHg}$, EVLWi of $>10 \mathrm{~mL} / \mathrm{kg}$, and diagnosed as permeability pulmonary edema. Third, patients who were not administered sivelestat on Day 0 were excluded from the current analysis to exclude the possibility of immortal-time bias [58]. Thus, we could not investigate the effect of sivelestat on the late phase of ARDS.

\section{Conclusions}

Although there was no significant association between 28-day mortality and administration of sivelestat, the treatment may have the potential to increase the number of VFDs in ARDS patients with increased EVLW. Prospective randomized controlled studies are required to confirm the results of the current study.

\section{Additional file}

Additional file 1: Figure S1. Distribution of propensity scores in the unmatched, propensity score-matched, and propensity score inverse probability of treatment weighting (IPTW) groups.

\section{Abbreviations}

APACHE: Acute physiology and chronic health evaluation; ARDS: Acute respiratory distress syndrome; CVP: Central venous pressure; EVLW: Extravascular lung water; EVLWi: Extravascular lung water index; NE: Neutrophil elastase; PEEP: Positive end-expiratory pressure; PVPI: Pulmonary vascular permeability index; SOFA: Sequential organ failure assessment; Cl: Confidence interval.

\section{Competing interests}

Takashi Tagami, Nobuyuki Saito, and Shigeki Kushimoto received speaker honoraria from ONO Pharmaceutical Co and Tokibo Co., Ltd (import trader of the PiCCO system) for educational lectures at Japanese scientific meetings. The other authors declare no conflicts of interest. 


\section{Authors' contributions}

All authors conceived and designed the study, wrote the study protocol, and acquired the clinical data. TT was responsible for the statistical analyses, the first draft, and critical revision of the manuscript. All authors amended and commented on the manuscript and approved the final version.

\section{Acknowledgements}

We would like to acknowledge all of the institutions that participated in the PiCCO Pulmonary Edema study and their staff. We are grateful to Professor Hideo Yasunaga, MD, PhD (Department of Clinical Epidemiology and Health Economics, School of Public Health, The University of Tokyo) for assistance during the statistical review of this study.

\section{Author details}

${ }^{1}$ Department of Emergency and Critical Care Medicine, Nippon Medical School, 1-1-5 Sendagi, Bunkyo-ku, Tokyo 113-8603, Japan. ²Department of Clinical Epidemiology and Health Economics, School of Public Health, Graduate School of Medicine, The University of Tokyo, Tokyo, Japan. ${ }^{3}$ Department of Emergency and Critical Care Medicine, Aizu Chuo Hospital, Fukushima, Japan. ${ }^{4}$ Department of Emergency and Critical Care Medicine, Nara Medical University, Nara, Japan. ${ }^{5}$ Advanced Medical Emergency and Critical Care Center, Yamaguchi University Hospital, Yamaguchi, Japan. ${ }^{6}$ Department of Emergency and Critical Care Medicine, Tohoku University Hospital, Miyagi, Japan. ${ }^{7}$ Emergency and Critical Care Medical Center, Osaka City General Hospital, Osaka, Japan. ${ }^{8}$ Department of Emergency and Critical Care Medicine, Faculty of Medicine, Fukuoka University, Fukuoka, Japan. ${ }^{9}$ Division of Emergency and Critical Care Medicine, Department of Acute Medicine, Nihon University School of Medicine, Tokyo, Japan. ${ }^{10}$ Shock Trauma and Emergency Medical Center, Tokyo Medical and Dental University Hospital of Medicine, Tokyo, Japan. ${ }^{11}$ Department of Emergency and Critical Care Medicine, Nippon Medical School Chiba Hokusou Hospital, Chiba, Japan. ${ }^{12}$ Department of Emergency and Critical Care Medicine, Kurume University School of Medicine, Fukuoka, Japan. ${ }^{13}$ Critical Care Medicine, Jikei University School of Medicine, Tokyo, Japan. ${ }^{14}$ Department of Anesthesia and Intensive Care, Hiroshima City Hospital, Hiroshima, Japan. ${ }^{15}$ Advanced Emergency and Critical Care Center, Kansai Medical University Takii Hospital, Osaka, Japan. ${ }^{16}$ Intensive Care Unit, Nagasaki University Hospital, Nagasaki, Japan. ${ }^{17}$ Intensive Care Unit, Kobe City Medical Center General Hospital, Hyogo, Japan. ${ }^{18}$ Department of Emergency and Critical Care Medicine, Kansai Medical University, Osaka, Japan. ${ }^{19}$ Department of Emergency and Critical Care Medicine, Juntendo University Nerima Hospital, Tokyo, Japan. ${ }^{20}$ Department of Intensive Care Medicine, Saiseikai Yokohamashi Tobu Hospital, Kanagawa, Japan. ${ }^{21}$ Department of Emergency and Critical Care Medicine, Social Insurance Chukyo Hospital, Aichi, Japan. ${ }^{22}$ Department of Emergency and Critical Care Medicine, Nippon Medical School Tama Nagayama Hospital, Tokyo, Japan. ${ }^{23}$ Emergency and Critical Care Medicine, National Hospital Organization Disaster Medical Center, Tokyo, Japan. ${ }^{24}$ Division of Emergency Medicine, Tohoku University Graduate School of Medicine, Miyagi, Japan.

Received: 10 July 2014 Accepted: 8 December 2014 Published online: 31 December 2014

\section{References}

1. Fujishima S: Pathophysiology and biomarkers of acute respiratory distress syndrome. J Intensive Care 2014, 2:32.

2. Koh Y: Update in acute respiratory distress syndrome. J Intensive Care 2014, 2:2.

3. Ranieri VM, Rubenfeld GD, Thompson BT, Ferguson ND, Caldwell E, Fan E, Camporota L, Slutsky AS: Acute respiratory distress syndrome: the Berlin definition. JAMA 2012, 307:2526-2533.

4. Zeiher BG, Matsuoka S, Kawabata K, Repine JE: Neutrophil elastase and acute lung injury: prospects for sivelestat and other neutrophil elastase inhibitors as therapeutics. Crit Care Med 2002, 30:S281-S287.

5. Donnelly SC, MacGregor I, Zamani A, Gordon MW, Robertson CE, Steedman DJ, Little K, Haslett C: Plasma elastase levels and the development of the adult respiratory distress syndrome. Am J Respir Crit Care Med 1995, 151:1428-1433.

6. Iba T, Kidokoro A, Fukunaga M, Takuhiro K, Yoshikawa S, Sugimotoa K: Pretreatment of sivelestat sodium hydrate improves the lung microcirculation and alveolar damage in lipopolysaccharide-induced acute lung inflammation in hamsters. Shock 2006, 26:95-98.

7. Tamakuma N, Shiba T, Hirasawa H, Ogawa M, Nakashima S: A phase III clinical study of neutrophil elastase inhibitor ONO-5046* Na in SIRS patients. J Clin Ther Med (Jpn) 1998, 14:289-318.

8. Zeiher BG, Artigas A, Vincent J-L, Dmitrienko A, Jackson K, Thompson BT, Bernard G: Neutrophil elastase inhibition in acute lung injury: results of the STRIVE study. Crit Care Med 2004, 32:1695-1702.

9. Tamakuma S, Ogawa M, Aikawa N, Kubota T, Hirasawa H, Ishizaka A, Taenaka N, Hamada C, Matsuoka S, Abiru T: Relationship between neutrophil elastase and acute lung injury in humans. Pulm Pharmacol Ther 2004, 17:271-279.

10. Aikawa N, Ishizaka A, Hirasawa H, Shimazaki S, Yamamoto $Y$, Sugimoto H, Shinozaki M, Taenaka N, Endo S, Ikeda T, Kawasaki Y: Reevaluation of the efficacy and safety of the neutrophil elastase inhibitor, Sivelestat, for the treatment of acute lung injury associated with systemic inflammatory response syndrome; a phase IV study. Pulm Pharmacol Ther 2011, 24:549-554.

11. Iwata K, Doi A, Ohji G, Oka H, Oba Y, Takimoto K, Igarashi W, Gremillion DH, Shimada T: Effect of neutrophil elastase inhibitor (sivelestat sodium) in the treatment of acute lung injury (ALI) and acute respiratory distress syndrome (ARDS): a systematic review and meta-analysis. Intern Med 2010, 49:2423-2432.

12. Oda S, Aibiki M, Ikeda T, Imaizumi H, Endo S, Ochiai R, Kotani J, Shime N, Nishida O, Noguchi T, Matuda N, Hirasawa H, Sepsis Registry Committee of JSICM: The Japanese guidelines for the management of sepsis. J Intensive Care 2014, 2:55.

13. Corrin B, Nicholson A: Pathology of the Lungs. Edinburgh: Churchill Livingstone; 2011.

14. Tagami T, Kushimoto S, Yokota H: Quantitative evaluation of pulmonary edema. In Annual Update in Intensive Care and Emergency Medicine 2014. Edited by Vincent J-L. Springer International Publishing; 2014: 257-267. [Vincent J-L (Series Editor)].

15. Jozwiak M, Silva S, Persichini R, Anguel N, Osman D, Richard C, Teboul JL, Monnet $X$ : Extravascular lung water is an independent prognostic factor in patients with acute respiratory distress syndrome. Crit Care Med 2013, 41:472-480

16. Kushimoto S, Endo T, Yamanouchi S, Sakamoto T, Ishikura H, Kitazawa Y, Taira Y, Okuchi K, Tagami T, Watanabe A, Yamaguchi J, Yoshikawa K, Sugita M, Kase Y, Kanemura T, Takahashi H, Kuroki Y, Izumino H, Rinka H, Seo R, Takatori M, Kaneko T, Nakamura T, Irahara T, Saito N, The PiCCO Pulmonary Edema Study Group: Relationship between extravascular lung water and severity categories of acute respiratory distress syndrome by the Berlin definition. Crit Care 2013, 17:R132.

17. Tagami T, Nakamura T, Kushimoto $S$, Tosa R, Watanabe A, Kaneko T, Fukushima H, Rinka H, Kudo D, Uzu H, Murai A, Takatori M, Izumino H, Kase Y, Seo R, Takahashi H, Kitazawa Y, Yamaguchi J, Sugita M, Takahashi H, Kuroki Y, Kanemura T, Morisawa K, Saito N, Irahara T, Yokota H: Early-phase changes of extravascular lung water index as a prognostic indicator in acute respiratory distress syndrome patients. Ann Intens Care 2014, 4:27.

18. Tagami T, Sawabe M, Kushimoto S, Marik PE, Mieno MN, Kawaguchi T, Kusakabe T, Tosa R, Yokota H, Fukuda Y: Quantitative diagnosis of diffuse alveolar damage using extravascular lung water. Crit Care Med 2013, 41:2144-2150.

19. Kushimoto S, Taira Y, Kitazawa Y, Okuchi K, Sakamoto T, Ishikura H, Endo T, Yamanouchi S, Tagami T, Yamaguchi J, Yoshikawa K, Sugita M, Kase Y, Kanemura T, Takahashi H, Kuroki Y, Izumino H, Rinka H, Seo R, Takatori M, Kaneko T, Nakamura T, Irahara T, Saito N, Watanabe A, The PiCCO Pulmonary Edema Study Group: The clinical usefulness of extravascular lung water and pulmonary vascular permeability index to diagnose and characterize pulmonary edema: a prospective multicenter study on the quantitative differential diagnostic definition for acute lung injury/acute respiratory distress syndrome. Crit Care 2012, 16:R232.

20. Endo T, Kushimoto S, Yamanouchi S, Sakamoto T, Ishikura H, Kitazawa Y, Taira Y, Okuchi K, Tagami T, Watanabe A, Yamaguchi J, Yoshikawa K, Sugita M, Kase Y, Kanemura T, Takahashi H, Kuroki Y, Izumino H, Rinka H, Seo R, Takatori M, Kaneko T, Nakamura T, Irahara T, Saito N: Limitations of global end-diastolic volume index as a parameter of cardiac preload in the early phase of severe sepsis: a subgroup analysis of a multicenter, prospective observational study. J Intens Care 2013, 1:11. 
21. Kaneko T, Kawamura Y, Maekawa T, Tagami T, Nakamura T, Saito N, Kitazawa Y, Ishikura H, Sugita M, Okuchi K, Rinka H, Watanabe A, Kase Y, Kushimoto S, Izumino H, Kanemura T, Yoshikawa K, Takahashi H, Irahara T, Sakamoto T, Kuroki Y, Taira Y, Seo R, Yamaguchi J, Takatori M: Global end-diastolic volume is an important contributor to increased extravascular lung water in patients with acute lung injury and acuterespiratory distress syndrome: a multicenter observational study. J Intens Care 2014, 2:25.

22. Morisawa K, Fujitani S, Taira Y, Kushimoto S, Kitazawa Y, Okuchi K, Ishikura H, Sakamoto T, Tagami T, Yamaguchi J, Sugita M, Kase Y, Kanemura T, Takahashi H, Kuroki Y, Izumino H, Rinka H, Seo R, Takatori M, Kaneko T, Nakamura T, Irahara T, Saitou N, Watanabe A: Difference in pulmonary permeability between indirect and direct acute respiratory distress syndrome assessed by the transpulmonary thermodilution technique: a prospective, observational, multi-institutional study. J Intens Care 2014, 2:24.

23. Knaus WA, Draper EA, Wagner DP, Zimmerman JE: APACHE II: a severity of disease classification system. Crit Care Med 1985, 13:818-829.

24. Ferreira FL, Bota DP, Bross A, Melot C, Vincent $J$ L: Serial evaluation of the SOFA score to predict outcome in critically ill patients. JAMA 2001, 286:1754-1758.

25. Monnet X, Anguel N, Osman D, Hamzaoui O, Richard C, Teboul JL: Assessing pulmonary permeability by transpulmonary thermodilution allows differentiation of hydrostatic pulmonary edema from ALI/ARDS. Intens Care Med 2007, 33:448-453.

26. Tagami T, Kushimoto S, Tosa R, Omura M, Yonezawa K, Akiyama G, Hirama $\mathrm{H}$, Yokota $\mathrm{H}$ : Plasma neutrophil elastase correlates with pulmonary vascular permeability: a prospective observational study in patients with pneumonia. Respirology 2011, 16:953-958.

27. Tagami T, Kushimoto S, Yamamoto Y, Atsumi T, Tosa R, Matsuda K, Oyama R, Kawaguchi T, Masuno T, Hirama H, Yokota $H$ : Validation of extravascular lung water measurement by single transpulmonary thermodilution: human autopsy study. Crit Care 2010, 14:R162.

28. Craig TR, Duffy MJ, Shyamsundar M, McDowell C, McLaughlin B, Elborn JS, McAuley DF: Extravascular lung water indexed to predicted body weight is a novel predictor of intensive care unit mortality in patients with acute lung injury. Crit Care Med 2010, 38:114-120.

29. Phillips CR, Chesnutt MS, Smith SM: Extravascular lung water in sepsisassociated acute respiratory distress syndrome: indexing with predicted body weight improves correlation with severity of illness and survival. Crit Care Med 2008, 36:69-73.

30. Schoenfeld DA, Bernard GR: Statistical evaluation of ventilator-free days as an efficacy measure in clinical trials of treatments for acute respiratory distress syndrome. Crit Care Med 2002, 30:1772-1777.

31. Austin PC: The use of propensity score methods with survival or time-toevent outcomes: reporting measures of effect similar to those used in randomized experiments. Stat Med 2014, 33:1242-1258.

32. Austin PC: An introduction to propensity score methods for reducing the effects of confounding in observational studies. Multivar Behav Res 2011, 46:399-424.

33. Mikkelsen ME, Shah CV, Meyer NJ, Gaieski DF, Lyon S, Miltiades AN, Goyal M, Fuchs BD, Bellamy SL, Christie JD: The epidemiology of acute respiratory distress syndrome in patients presenting to the emergency department with severe sepsis. Shock 2013, 40:375-381.

34. Lin C-Y, Kao K-C, Tian Y-C, Jeng C-C, Chang M-Y, Chen Y-C, Fang J-T, Huang C-C, Tsai Y-H, Yang C-W: Outcome scoring systems for acute respiratory distress syndrome. Shock 2010, 34:352-357.

35. Hayashida K, Fujishima S, Sasao K, Orita T, Toyoda Y, Kitano M, Hori S: Early administration of sivelestat, the neutrophil elastase inhibitor, in adults for acute lung injury following gastric aspiration. Shock 2011, 36:223-227.

36. Hayakawa M, Katabami K, Wada T, Sugano M, Hoshino H, Sawamura A Gando S: Sivelestat (selective neutrophil elastase inhibitor) improves the mortality rate of sepsis associated with both acute respiratory distress syndrome and disseminated intravascular coagulation patients. Shock 2010, 33:14-18.

37. Hashimoto S, Okayama Y, Shime N, Kimura A, Funakoshi Y, Kawabata K, Ishizaka A, Amaya F: Neutrophil elastase activity in acute lung injury and respiratory distress syndrome. Respirology 2008, 13:581-584.

38. Endo S, Sato N, Yaegashi Y, Suzuki Y, Kojika M, Yamada Y, Yoshida Y, Nakadate T, Aoki H, Inoue Y: Sivelestat sodium hydrate improves septic acute lung injury by reducing alveolar dysfunction. Res Commun $\mathrm{Mol}$ Pathol Pharmacol 2006, 119:53-65.
39. Takeda S, Ishizaka A, Fujino Y, Fukuoka T, Nagano O, Yamada Y, Takezawa J: Time to change diagnostic criteria of ARDS: toward the disease entitybased subgrouping. Pulm Pharmacol Ther 2005, 18:115-119.

40. Iwata K: The propensity score analysis on the efficacy of sivelestat. Pulm Pharmacol Ther 2013, 26:395.

41. Aikawa N: The propensity score analysis on the efficacy of Sivelestat author's reply. Pulm Pharmacol Ther 2013, 26:396-397.

42. Rinka H, Yoshimoto A, Matsuura Y, Miyaichi T, Kan A, Kaji A, Miyanmoto S: Neutrophil elastase inhibitor improves the vascular permeability in patients with sepsis induced ALI/ARDS. Nihon Kyukyu lgakukai Zasshi 2007, 7:283-290. (Japanese).

43. Rubenfeld GD, Caldwell E, Peabody E, Weaver J, Martin DP, Neff M, Stern EJ, Hudson LD: Incidence and outcomes of acute lung injury. N Engl J Med 2005, 353:1685-1693.

44. Cruz DN, Antonelli M, Fumagalli R, Foltran F, Brienza N, Donati A, Malcangi V, Petrini F, Volta G, Bobbio Pallavicini FM, Rottoli F, Giunta F, Ronco C: Early use of polymyxin $B$ hemoperfusion in abdominal septic shock: the EUPHAS randomized controlled trial. JAMA 2009, 301:2445-2452.

45. Lee SK, Son BS, Hwang JJ, Kim KD, Kim DH: The use of neutrophil elastase inhibitor in the treatment of acute lung injury after pneumonectomy. J Cardiothorac Surg 2013, 8:69.

46. Tagami T, Matsui H, Horiguchi H, Fushimi K, Yasunaga H: Low-dose corticosteroid use and mortality in severe community-acquired pneumonia patients. Eur Resp J 2014. In Press.

47. Tsuboko Y, Takeda S, Sakamoto A, Mii S, Nakazato K, Tanaka K, Uchida E: Clinical evaluation of sivelestat for acute lung injury/acute respiratory distress syndrome following surgery for abdominal sepsis. Drug Des Devel Ther 2012, 6:273-278.

48. Miyoshi S, Hamada H, Ryoji I, Katayama H, Irifune K, Suwaki T, Nakanishi N, Kanematsu T, Kentaro D, Aibiki M, Okura T, Higaki J: Usefulness of a selective neutrophil elastase inhibitor, sivelestat, in acute lung injury patients with sepsis. Drug Des Devel Ther 2013, 7:305-316.

49. Tang BM, Craig JC, Eslick GD, Seppelt I, McLean AS: Use of corticosteroids in acute lung injury and acute respiratory distress syndrome: a systematic review and meta-analysis. Crit Care Med 2009, 37:1594-1603.

50. Tagami T, Kuwamoto K, Watanabe A, Unemoto K, Yokobori S, Matsumoto G, Yokota H, Group SAHPS: Optimal range of global end-diastolic volume for fluid management after aneurysmal subarachnoid hemorrhage: a multicenter prospective cohort study. Crit Care Med 2014, 42:1348-1356.

51. Kadoi Y, Hinohara H, Kunimoto F, Saito S, Goto F, Kosaka T, Leta K: Pilot study of the effects of ONO-5046 in patients with acute respiratory distress syndrome. Anesth Analg 2004, 99:872-877.

52. Bernard GR, Artigas A, Brigham KL, Carlet J, Falke K, Hudson L, Lamy M, Legall JR, Morris A, Spragg R: The American-European Consensus Conference on ARDS. Definitions, mechanisms, relevant outcomes, and clinical trial coordination. Am J Respir Crit Care Med 1994, 149:818-824.

53. Monnet X, Persichini R, Ktari M, Jozwiak M, Richard C, Teboul JL: Precision of the transpulmonary thermodilution measurements. Crit Care 2011, 15:R204.

54. Michard F, Fernandez-Mondejar E, Kirov MY, Malbrain M, Tagami T: A new and simple definition for acute lung injury. Crit Care Med 2012, 40:1004-1006.

55. Martin GS, Eaton S, Mealer M, Moss M: Extravascular lung water in patients with severe sepsis: a prospective cohort study. Crit Care 2005, 9:R74-R82.

56. Kuzkov W, Kirov MY, Sovershaev MA, Kuklin VN, Suborov EV, Waerhaug K, Bjertnaes LJ: Extravascular lung water determined with single transpulmonary thermodilution correlates with the severity of sepsisinduced acute lung injury. Crit Care Med 2006, 34:1647-1653.

57. Letourneau JL, Pinney J, Phillips CR: Extravascular lung water predicts progression to acute lung injury in patients with increased risk. Crit Care Med 2012, 40:847-854.

58. Suissa S: Immortal time bias in pharmaco-epidemiology. Am J Epidemiol 2008, 167:492-499. 